\title{
CAN FERRITIN / LYMPHOCYTE PERCENTAGE RATIO, A NEW INDICATOR, PREDICT THE CLINICAL COURSE OF COVID-19 CASES?
}

\author{
Huseyin AYGUN ${ }^{1}$ and Suna ERAYBAR ${ }^{1}$ \\ ${ }^{1}$ Bursa Yuksek Ihtisas Training and Research Hospital
}

February 22, 2021

\begin{abstract}
Aim: Covid-19 maintains its seriousness as a global emergency with its rapid distribution worldwide. Ferritin / lymphocyte percentage ratio (FLPR) may appear as a prognostic value at the initial evaluation stage and thus can be used as a simple, effective and reliable parameter in critical patient identification with Covid-19. Materials and Methods: In this retrospective cohort study we evaluated patients over 18 years old, who were hospitalized after being evaluated as COVID-19 and whose PCR results were positive. We calculated FLPRs from complete blood counts taken during emergency department admissions and classified disease severity due to emergency initial evaluation. The relationship between the severity of the thoracic tomography findings, hospitalization and intensive care needs, and 28-day mortality with the FLPR were evaluated. Results: The difference between the groups classified according to COVID-19 severity and the FLPR means was statistically significant (x2=148,284; $\mathrm{SD}=3 ; \mathrm{p}=0,000)$. FLPR levels were found to be high in critical and serious groups. In the ROC analysis for the FLPR level, the area under the curve (AUC) value was found to be 0.909 (95\% CI 0.857-0.961). When the cut off value of FLPR was 9.80, the sensitivity was found to be $97.6 \%$, and the specificity was $65.2 \%$, whereas, when the cut off value for FLPR was found to be 21.11 , the sensitivity was $82.9 \%$ and the specificity was $82.8 \%$. Conclusion: The FLPR, a new parameter, can be used as a significant marker to predict the 28-day mortality patients.
\end{abstract}

\section{Hosted file}

ICP FERR \selectlanguage\{polish\} $\dot{I} \backslash$ selectlanguage english\}T $\backslash$ selectlanguage $\{$ polish $\} \dot{I} \backslash$ selectlanguage $\{$ englis available at https://authorea.com/users/397256/articles/510194-can-ferritin-lymphocytepercentage-ratio-a-new-indicator-predict-the-clinical-course-of-covid-19-cases 